2013

\title{
Calculation Of Non-Adiabatic Coupling Vectors In A Local-Orbital Basis Set
}

\author{
Enrique Abad
}

James P. Lewis

Vladmír Zobač

Prokop Hapala

Pavel Jelínek

See next page for additional authors

Follow this and additional works at: https://researchrepository.wvu.edu/faculty_publications

\section{Digital Commons Citation}

Abad, Enrique; Lewis, James P.; Zobač, Vladmír; Hapala, Prokop; Jelínek, Pavel; and Ortega, José, "Calculation Of Non-Adiabatic Coupling Vectors In A Local-Orbital Basis Set" (2013). Faculty Scholarship. 479.

https://researchrepository.wvu.edu/faculty_publications/479 
Authors

Enrique Abad, James P. Lewis, Vladmír Zobač, Prokop Hapala, Pavel Jelínek, and José Ortega 


\title{
Calculation of non-adiabatic coupling vectors in a local-orbital basis set
}

\author{
Enrique Abad, ${ }^{1}$ James P. Lewis, ${ }^{2}$ Vladmír Zobač, ${ }^{3}$ Prokop Hapala, ${ }^{3}$ Pavel Jelínek, ${ }^{3}$ \\ and José Ortega ${ }^{1, a)}$ \\ ${ }^{1}$ Departamento de Física Teórica de la Materia Condensada and Condensed Matter Physics Center (IFIMAC), \\ Universidad Autónoma de Madrid, ES-28049 Madrid, Spain \\ ${ }^{2}$ Department of Physics, West Virginia University, Morgantown, West Virginia 26506-6315, USA \\ ${ }^{3}$ Institute of Physics, Academy of Sciences of the Czech Republic, Cukrovarnická 10, CZ-16200 Prague, \\ Czech Republic
}

(Received 12 January 2013; accepted 26 March 2013; published online 18 April 2013)

\begin{abstract}
Most of today's molecular-dynamics simulations of materials are based on the Born-Oppenheimer approximation. There are many cases, however, in which the coupling of the electrons and nuclei is important and it is necessary to go beyond the Born-Oppenheimer approximation. In these methods, the non-adiabatic coupling vectors are fundamental since they represent the link between the classical atomic motion of the nuclei and the time evolution of the quantum electronic state. In this paper we analyze the calculation of non-adiabatic coupling vectors in a basis set of local orbitals and derive an expression to calculate them in a practical and computationally efficient way. Some examples of the application of this expression using a local-orbital density functional theory approach are presented for a few simple molecules: $\mathrm{H}_{3}$, formaldimine, and azobenzene. These results show that the approach presented here, using the Slater transition-state density, is a very promising way for the practical calculation of non-adiabatic coupling vectors for large systems. (C) 2013 AIP Publishing LLC. [http://dx.doi.org/10.1063/1.4801511]
\end{abstract}

\section{INTRODUCTION}

First-principles molecular-dynamics (FPMD) simulations have become a very powerful tool to understand the properties of materials as these techniques provide direct access to the dynamical and electronic processes that take place at the atomic scale. FPMD simulations are a perfect complement to the experimental techniques because they can probe shorter time- and length-scale that are not always observable from experiment. With continual improvements of FPMD simulation techniques, together with increasing computational power, these computational "atomic microscopes" can be applied to increasingly complex problems, involving larger unit-cells and longer simulation times.

The vast majority of FPMD simulations are based on density functional theory (DFT) $)^{1,2}$ and the adiabatic (or BornOppenheimer) approximation. In this approximation the nuclei move in a potential energy surface (PES) defined by the ground state energy of the electronic problem with fixed atomic positions. Thus, in this adiabatic approximation nuclei and electrons are decoupled and the nuclear motion does not change the quantum state of the electronic subsystem. Although this approach is very useful and has been widely employed to a large variety of systems, there are many important problems in physics, chemistry, biology, etc., that require to go beyond the adiabatic approximation (e.g., see Refs. 3-6). Some illustrative examples are: photochemical processes in biology and chemistry (photosynthesis, photocatalysis, etc.), radiationless decay of excited states (i.e., internal conversion), material damage by high energy particles or by ra-

\footnotetext{
a)Electronic mail: jose.ortega@uam.es
}

diation, Joule heating, ion-surface collisions, non-adiabatic charge transfer, etc. In general, those phenomena where the coupling of electrons and nuclei is relevant, cannot be described properly using the adiabatic approximation.

The non-adiabatic coupling vectors (NACVs) play a fundamental role in order to extend FPMD simulations beyond the adiabatic approximation since they provide the link between the classical atomic motion and the change of the quantum electronic state. ${ }^{7-14}$ Most of the interesting applications of non-adiabatic FPMD involve the simulation of large and complex systems (e.g., biomolecules). ${ }^{15-17}$ Thus, it is necessary to devise efficient methods to target practical applications with several hundred or potentially thousands of atoms involved. In this work we analyze the calculation of NACVs in a basis set of local-orbitals and derive an expression that allows the direct calculation of NACVs in a practical and computationally efficient way, using information (matrix elements between local orbitals) that can be stored in pre-calculated datatables. ${ }^{18}$ In most of the applications of non-adiabatic FPMD, the NACVs are not calculated explicitly; instead, these methods use the non-adiabatic coupling elements (scalar product of the NACVs with the atomic velocities, see Eq. (5)) since they are easily obtained as a numerical finite difference formula. ${ }^{19,20}$ However, it is both interesting and useful to explicitly calculate the NACVs for instance, in order to search for conical intersections or avoided crossing regions, ${ }^{21-24}$ or for the rescaling of the atomic velocities when an electronic transition takes place along the non-adiabatic molecular dynamics (MD) simulation. . $^{85-27}$

In Sec. II, we review the basic theory for non-adiabatic MD as well as its implementation in the framework of local-orbital DFT (in particular our local-orbital DFT MD 
technique FIREBALL ${ }^{18,28}$ ). In Sec. III we present the derivation of a practical expression for the calculation of the NACVs in a local-orbital basis set; all the derivatives (with respect to atomic displacements) that appear in this expression can be obtained from pre-calculated data-tables, allowing a computationally efficient calculation of the NACVs on the fly along the MD simulation. From this expression we also derive an approximate expression that yields the NACVs in terms of the derivatives of the matrix elements of the Hamiltonian and the overlap matrix in the local-orbital basis which is especially suited for tight-binding MD methods. Finally, in Sec. IV, we present test calculations of the NACVs for some simple molecules: $\mathrm{H}_{3}$, formaldimine, and azobenzene.

\section{THEORETICAL BACKGROUND}

In non-adiabatic MD simulations, the atoms follow classical trajectories $\left\{\mathbf{R}_{\alpha}(t)\right\}$, but the inter-relation between nuclear motion and electronic quantum state is considered throughout the simulation. For this purpose, the time-evolution of the electronic wavefunction is explicitly considered by means of the time-dependent Schrödinger equation:

$$
\hat{H}_{e l} \tilde{\Psi}=i \hbar \frac{\partial \tilde{\Psi}}{\partial t}
$$

where $\hat{H}_{e l}$ is the electronic Hamiltonian for fixed atomic positions (i.e., the total Hamiltonian minus the kinetic energy of the nuclei). It is convenient to express the electronic wavefunction $\tilde{\Psi}$ in the basis set of instantaneous adiabatic eigenstates $\Psi_{i}(\mathbf{r}, \mathbf{R})$ which are eigenstates of $\hat{H}_{e l}$, i.e., solution of the stationary Schrödinger equation with fixed atomic positions, $\left\{\mathbf{R}_{\alpha}\right\}$ :

$$
\begin{gathered}
\tilde{\Psi}(\mathbf{r}, t)=\sum_{i} a_{i}(t) \Psi_{i}(\mathbf{r}, \mathbf{R}), \\
\hat{H}_{e l} \Psi_{i}(\mathbf{r}, \mathbf{R})=E_{i}(\mathbf{R}) \Psi_{i}(\mathbf{r}, \mathbf{R}),
\end{gathered}
$$

where $\mathbf{r}$ stands for all the electron coordinates and $\mathbf{R}$ represents the atomic positions $\left\{\mathbf{R}_{\alpha}(t)\right\}$ at time $t$. Introducing these expressions in Eq. (1) we can obtain the time-evolution for the electronic wavefunction $\tilde{\Psi}(\mathbf{r}, t)$ through the time evolution of the coefficients $a_{i}(t)$ :

$$
i \hbar \frac{\partial a_{i}(t)}{\partial t}=a_{i}(t) E_{i}(\mathbf{R})-i \hbar \sum_{j} a_{j}(t) \mathbf{D}_{i j} \cdot \mathbf{V}
$$

In this equation, the coupling between the classical motion of the atoms and the electronic quantum state is reflected in the non-adiabatic coupling term, $\mathbf{D}_{i j} \cdot \mathbf{V}$ :

$$
\mathbf{D}_{i j} \cdot \mathbf{V} \equiv \sum_{\alpha} \mathbf{D}_{i j}^{\alpha} \mathbf{V}_{\alpha}
$$

where $\mathbf{V}_{\alpha}=\partial \mathbf{R}_{\alpha} / \partial t$ is the atomic velocity of atom $\alpha$ and $\mathbf{D}_{i j}^{\alpha}$ are the non-adiabatic coupling vectors:

$$
\mathbf{D}_{i j}^{\alpha} \equiv\left\langle\Psi_{i} \mid \frac{\partial \Psi_{j}}{\partial \mathbf{R}_{\alpha}}\right\rangle .
$$

Thus, in Eq. (4), the atomic motions, $\mathbf{V}_{\alpha}$, induce changes in the adiabatic state populations $\left|a_{i}(t)\right|^{2}$ through their coupling with the non-adiabatic coupling vectors, $\mathbf{D}_{i j}^{\alpha}$.

In practice, the quantum mechanical problem, Eqs. (1)(3), is solved using single particle orbitals (e.g., Hartree-Fock (HF) or Kohn-Sham (KS) orbitals). These single-particle orbitals can be used to calculate the different PES: the HF or KS ground states are related to the Slater determinants formed with the set of occupied lowest-energy HF or KS-orbitals, and excited states can also be obtained from similar Slater determinants, e.g., as linear combinations of singly excited Slater determinants. The many-body NACVs, $\mathbf{D}_{i j}^{\alpha}$ (Eq. (6)), are thus related to their corresponding single-particle NACVs, $\mathbf{d}_{i j}^{\alpha}$ (Eq. (11)). ${ }^{12,20}$

In time-dependent Kohn-Sham theory, ${ }^{29}$ the time evolution of the single-particle $\mathrm{KS}$ orbitals is obtained from the time-dependent Schrödinger-like equation:

$$
\hat{H}_{K S} \varphi_{p}=i \hbar \frac{\partial \varphi_{p}}{\partial t}
$$

where $\hat{H}_{K S}$ is the single-particle KS Hamiltonian and $\varphi_{p}(\mathbf{r}, t)$ are the time-evolving KS orbitals. In similarity to Eqs. (2) and (3), the orbitals $\varphi_{p}(\mathbf{r}, t)$ can be expressed in terms of the adiabatic KS orbitals, $\psi_{i}(\mathbf{r}, \mathbf{R})$ :

$$
\begin{gathered}
\hat{H}_{K S}(\mathbf{R}) \psi_{i}=\varepsilon_{i}(\mathbf{R}) \psi_{i}, \\
\varphi_{p}(\mathbf{r}, t)=\sum_{i} a_{i}(t) \psi_{i}(\mathbf{r}, \mathbf{R}) .
\end{gathered}
$$

The coefficients $a_{i}(t)$ in Eq. (9) are now calculated from the equations

$$
i \hbar \frac{\partial a_{i}(t)}{\partial t}=a_{i}(t) \varepsilon_{i}(\mathbf{R})-i \hbar \sum_{j} a_{j}(t) \mathbf{d}_{i j} \cdot \mathbf{V},
$$

$\left(\mathbf{d}_{i j} \cdot \mathbf{V} \equiv \sum_{\alpha} \mathbf{d}_{i j}^{\alpha} \mathbf{V}_{\alpha}\right)$, where $\mathbf{d}_{i j}^{\alpha}$ are the non-adiabatic coupling vectors between single-particle KS states

$$
\mathbf{d}_{i j}^{\alpha} \equiv\left\langle\psi_{i} \mid \frac{\partial \psi_{j}}{\partial \mathbf{R}_{\alpha}}\right\rangle .
$$

Broadly speaking, two types of non-adiabatic MD methods dominate the literature: Ehrenfest dynamics methods (see e.g., Refs. 3, 5, and 30-32) and surface hopping methods. ${ }^{19,20,25,26,33-35}$ In the Ehrenfest approach, the nuclei move classically on a single effective PES obtained by averaging over all the adiabatic states involved, whereas in surface hopping methods, the classical degrees of freedom (nuclei coordinates) evolve on single adiabatic surfaces (PES), and make probabilistic hops from one PES to another; when a transition takes place, energy conservation is imposed re-scaling velocities along the direction of the nonadiabatic coupling vectors ${ }^{8,25,27}$ (if the NACVs are not available, as is usually the case, energy conservation is achieved re-scaling all velocities). Surface hopping methods present well-known advantages over Ehrenfest methods (e.g., see Ref. 4). The surface hopping method has been implemented within $\mathrm{DFT}^{20,33,34}$ for calculations of systems on the order of 100 atoms and with durations of up to a few picoseconds. In particular, surface hopping simulations using a 
time-dependent KS (TDKS) approach ${ }^{20}$ have been used to analyze non-adiabatic dynamics in a number of relatively large systems; ${ }^{42-44}$ this TDKS approach ${ }^{20}$ has been compared with the more accurate linear-response time-dependent DFT (LRTDDFT) method $^{34}$ by performing non-adiabatic simulations for different systems. ${ }^{45}$ The results show that the simpler and computationally more efficient TDKS approach yields a very reasonable description of the systems analyzed, the agreement with the more accurate method being better for larger systems. $^{45}$

For practical applications to complex systems, it is very important to use non-adiabatic simulation techniques with a high computational efficiency. Some examples are ultrafast excited state dynamics in nucleic acids, ${ }^{36}$ energy transfer in photosynthetic proteins, ${ }^{37}$ non-adiabatic molecule/surface processes, ${ }^{38}$ etc. As we would like to devise non-adiabatic methods to target practical applications with several hundred or potentially thousands of atoms involved, we are developing a non-adiabatic MD method based on the local-orbital DFT MD technique FIREBALL. ${ }^{18,28}$ In this real-space implementation of DFT, numerical atomic-like orbitals are used as basis set. An important feature of this MD technique is that the different contributions to the KS Hamiltonian matrix elements can be calculated beforehand and tabulated in datatables. Thus, all the information required during the MD simulation is quickly obtained from this data-tables, speeding up the calculations (see Refs. 18 and 28 for details).

The discussion presented in this section highlights the importance of developing algorithms for the efficient calculation of the NACVs on the fly along a MD run. As mentioned above, the NACVs are used in surface hopping methods for velocity re-scaling (or hop rejection) when a transition is predicted, resulting in detailed balance and correct equilibrium behavior. ${ }^{20,39}$ Also, those algorithms are important in the search for conical intersections or avoided crossing regions, ${ }^{21-24}$ especially for large systems. In Sec. III we discuss how the NACVs, $\mathbf{d}_{i j}^{\alpha}$, can be calculated in a practical way within a local-orbital method. In particular, we derive an expression that allows a fast calculation of $\mathbf{d}_{i j}^{\alpha}$ for any geometry along the MD simulation, using only the eigenvalues $\varepsilon_{i}$ and expansion coefficients $c_{i \mu}$ (see below) of the single-particle states in the local-orbital basis, and pre-calculated information stored in data-tables.

\section{CALCULATION OF NON-ADIABATIC COUPLING VECTORS IN A LOCAL-ORBITAL BASIS SET}

We analyze here the calculation of NACVs between single-particle states within a local-orbital scheme. Although the derivation of Eqs. (18) and (19) is presented here for the particular case of the KS orbitals, we stress that this analysis, and the expressions obtained, are valid in general for any local-orbital method, such as semi-empirical tight-binding or Hartree-Fock-based methods.

We start by writing down the adiabatic KS states, $\psi_{i}$, in terms of the local-orbital basis set, $\phi_{\mu}$ :

$$
\psi_{i}=\sum_{\mu} c_{i \mu} \phi_{\mu}
$$

The NACVs between KS states in the local-orbital basis can be calculated as

$\mathbf{d}_{i j}^{\alpha}=\left\langle\psi_{i} \mid \frac{\partial \psi_{j}}{\partial \mathbf{R}_{\alpha}}\right\rangle=\sum_{\mu \nu} c_{i \mu}^{*} \frac{\partial c_{j v}}{\partial \mathbf{R}_{\alpha}} S_{\mu \nu}+\sum_{\mu \nu} c_{i \mu}^{*} c_{j v}\left\langle\phi_{\mu} \mid \frac{\partial \phi_{\nu}}{\partial \mathbf{R}_{\alpha}}\right\rangle$,

where $S_{\mu \nu}$ is the overlap matrix $S_{\mu \nu}=\left\langle\phi_{\mu} \mid \phi_{\nu}\right\rangle$. The practical problem with this expression is the calculation of the derivatives $\partial c_{j v} / \partial \mathbf{R}_{\alpha}$. In order to circumvent this problem, we use the identity

$$
\frac{\partial}{\partial \mathbf{R}_{\alpha}}\left\langle\psi_{i}\left|\hat{H}_{K S}\right| \psi_{j}\right\rangle=0,
$$

that yields the following relation:

$$
\begin{gathered}
\sum_{\mu \nu} c_{i \mu}^{*} c_{j v} \frac{\partial h_{\mu \nu}}{\partial \mathbf{R}_{\alpha}}+\sum_{\mu \nu} \frac{\partial c_{i \mu}^{*}}{\partial \mathbf{R}_{\alpha}} c_{j v} S_{\mu \nu} \varepsilon_{j} \\
+\sum_{\mu \nu} c_{i \mu}^{*} \frac{\partial c_{j v}}{\partial \mathbf{R}_{\alpha}} S_{\mu \nu} \varepsilon_{i}=0
\end{gathered}
$$

where $h_{\mu \nu}=\left\langle\phi_{\mu}\left|\hat{H}_{K S}\right| \phi_{\nu}\right\rangle$ are the KS Hamiltonian matrix elements in the local-orbital basis set. Using also the following relations:

$$
\left\langle\frac{\partial \psi_{i}}{\partial \mathbf{R}_{\alpha}} \mid \psi_{j}\right\rangle=\sum_{\mu \nu} \frac{\partial c_{i \mu}^{*}}{\partial \mathbf{R}_{\alpha}} c_{j v} S_{\mu \nu}+\sum_{\mu \nu} c_{i \mu}^{*} c_{j v}\left\langle\frac{\partial \phi_{\mu}}{\partial \mathbf{R}_{\alpha}} \mid \phi_{\nu}\right\rangle
$$

and

$$
\left\langle\frac{\partial \psi_{i}}{\partial \mathbf{R}_{\alpha}} \mid \psi_{j}\right\rangle=-\left\langle\psi_{i} \mid \frac{\partial \psi_{j}}{\partial \mathbf{R}_{\alpha}}\right\rangle
$$

we can finally obtain

$$
\begin{aligned}
\mathbf{d}_{i j}^{\alpha}= & \left\langle\psi_{i} \mid \frac{\partial \psi_{j}}{\partial \mathbf{R}_{\alpha}}\right\rangle=\frac{1}{\varepsilon_{i}-\varepsilon_{j}} \sum_{\mu \nu} c_{i \mu}^{*} c_{j v} \\
& \times\left[-\frac{\partial h_{\mu \nu}}{\partial \mathbf{R}_{\alpha}}+\varepsilon_{j}\left\langle\frac{\partial \phi_{\mu}}{\partial \mathbf{R}_{\alpha}} \mid \phi_{\nu}\right\rangle+\varepsilon_{i}\left\langle\phi_{\mu} \mid \frac{\partial \phi_{\nu}}{\partial \mathbf{R}_{\alpha}}\right\rangle\right] .
\end{aligned}
$$

This expression allows for the direct on-the-fly calculation of the NACVs $\mathbf{d}_{i j}^{\alpha}$. In Eq. (18) the NACVs are calculated using the $\mathrm{KS}$ eigenvalues, $\varepsilon_{i}$, $\varepsilon_{j}$, the expansion coefficients, $c_{i \mu}$, and the derivatives $\frac{\partial h_{\mu \nu}}{\partial \mathbf{R}_{\alpha}},\left\langle\frac{\partial \phi_{\mu}}{\partial \mathbf{R}_{\alpha}} \mid \phi_{\nu}\right\rangle$, and $\left\langle\phi_{\mu} \mid \frac{\partial \phi_{\nu}}{\partial \mathbf{R}_{\alpha}}\right\rangle$ (but $\left.\operatorname{not} \partial c_{j v} / \partial \mathbf{R}_{\alpha}\right)$. In particular, in our local-orbital DFT method (FIREBALL ${ }^{28}$ ) all the derivatives that appear in this expression can be obtained from pre-calculated information stored in data-tables, and thus the NACVs $\mathbf{d}_{i j}^{\alpha}$ can be calculated in a fast and practical way using Eq. (18).

Non-adiabatic effects are generally important only in regions near avoided crossings and conical intersections; in these regions $\varepsilon_{i} \approx \varepsilon_{j}$, and Eq. (18) can be approximated as

$$
\mathbf{d}_{i j}^{\alpha}=\frac{1}{\varepsilon_{i}-\varepsilon_{j}} \sum_{\mu \nu} c_{i \mu}^{*} c_{j v}\left[-\frac{\partial h_{\mu \nu}}{\partial \mathbf{R}_{\alpha}}+\frac{\left(\varepsilon_{j}+\varepsilon_{i}\right)}{2} \frac{\partial S_{\mu \nu}}{\partial \mathbf{R}_{\alpha}}\right] .
$$

Notice that the only derivatives that appear in Eq. (19) are the derivatives with respect to atomic displacements of the matrix elements of the KS Hamiltonian, $h_{\mu \nu}$, and overlap matrix, $S_{\mu \nu}$. In semi-empirical tight-binding MD methods, analytical expressions (or numerical pre-calculated data-tables) 

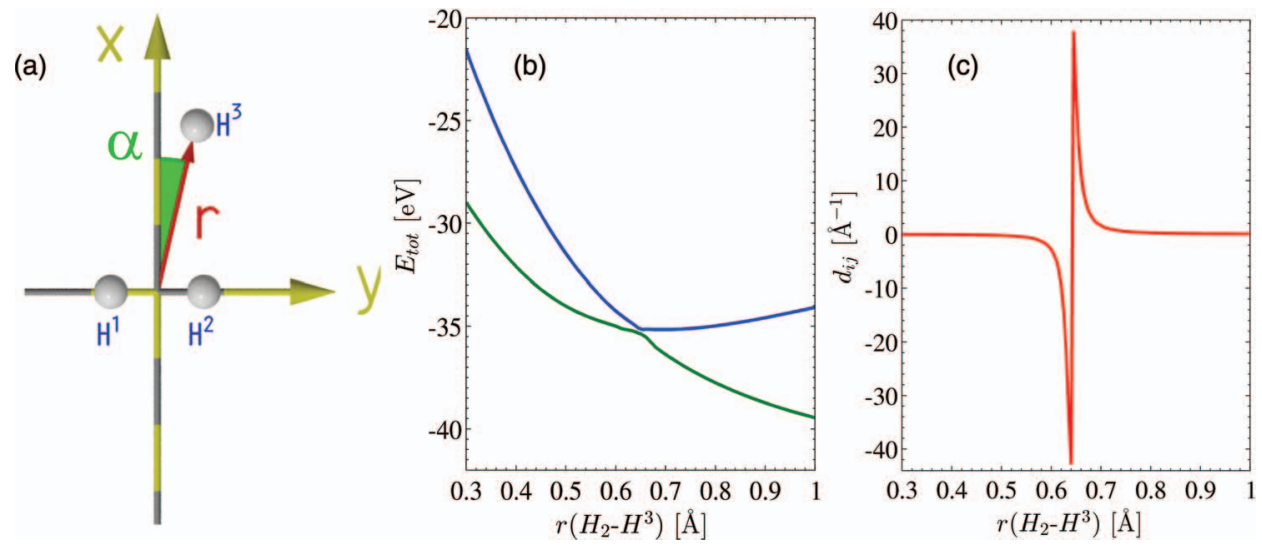

FIG. 1. (a) Schematic representation of the $\mathrm{H}_{3}$ system and the trajectory (along the vector $\mathbf{r}$ ) of the third $\mathrm{H}$ atom towards a fixed $\mathrm{H}_{2}$ molecule. This trajectory is defined by a small angle, $\alpha=1^{\circ}$, with respect to the X-axis. (b) PES for the ground ( $E_{0}$, green) and first excited $\left(E_{1}\right.$, blue) state of the $\mathrm{H}_{3}$ system as a function of the distance $r$ along this trajectory. (c) Evolution of the X-component of the non-adiabatic coupling vector $\mathbf{d}_{i j}^{\alpha}$ between the HOMO and LUMO KS states along the trajectory.

for $h_{\mu \nu}$ and $S_{\mu \nu}$ are used and the derivatives $\partial h_{\mu \nu} / \partial \mathbf{R}_{\alpha}$ and $\partial S_{\mu \nu} / \partial \mathbf{R}_{\alpha}$ are readily available. Thus, Eq. (19) is ideally suited for non-adiabatic MD techniques based on tightbinding MD, or similar approaches. ${ }^{40}$ Another interesting property of Eq. (19) is that it is completely translationally invariant. $^{13,14}$

\section{RESULTS}

In this section, we present some examples of the calculation of the non-adiabatic coupling vectors $\mathbf{d}_{i j}^{\alpha}$ using Eq. (18) for a few simple molecules, $H_{3}$, formaldimine, and azobenzene, showing how the NACVs between selected electronic states (HOMO and LUMO) vary upon conformational changes. For this purpose, we have implemented Eq. (18), in the local-orbital DFT technique FIREBALL. ${ }^{18,28}$ These test calculations have been performed using the local density approximation (LDA) for the exchange-correlation functional, ${ }^{2}$ and a basis set of numerical atomic-like orbitals ${ }^{18,41}$ these orbitals are completely localized in space, i.e., they are strictly zero beyond a given cutoff radius $R_{c} \cdot{ }^{18}$ In particular, we have used the following cutoff radii: $R_{c}^{H}(s)=3.8$ a.u. and $R_{c}^{H}\left(s^{*}\right)=3.8$ a.u. for $\mathrm{H} ; R_{c}^{C}(s)=4.5$ a.u., $R_{c}^{C}(p)=4.5$ a.u., and $R_{c}^{C}(d)=5.4$ a.u. for C; and $R_{c}^{N}(s)=3.6$ a.u, $R_{c}^{N}(p)$ $=4.1$ a.u., and $R_{c}^{N}(d)=5.2$ a.u. for $\mathrm{N}$.

The NACVs presented in the following examples correspond to the single-particle NACVs, $\mathbf{d}_{i j}^{\alpha}$, between HOMO and LUMO KS states. As mentioned in Sec. II, the NACVs $\mathbf{d}_{i j}^{\alpha}$ can be used to calculate the NACVs between many-body states, $\mathbf{D}_{i j}^{\alpha}$ (e.g., representing the excited states as linear combinations of excited Slater determinants). In this regards, the NACVs $\mathbf{d}_{i j}^{\alpha}$ between HOMO and LUMO KS states are the zeroth-order approximation to the $\mathbf{D}_{i j}^{\alpha}$ between ground and first excited states, in which the excited state is represented by a single excited Slater determinant. In particular, the NACVs between the HOMO and LUMO KS states from a Slater transition-state ${ }^{7,10}$ calculation yield a good approximation for the many-body NACVs between the ground state and the corresponding particle-hole excitation, see below.

\section{A. $\mathrm{H}_{3}$ system}

As a first example, we study the evolution of the nonadiabatic coupling vectors $\mathbf{d}_{i j}^{\alpha}$ during the hydrogen exchange reaction $\mathrm{H}+\mathrm{H}_{2} \rightarrow \mathrm{H}_{2}+\mathrm{H}$. The hydrogen exchange interaction is a prototype for bimolecular reactions. Therefore this process has been extensively studied using different theoretical approaches ${ }^{9,12,46-49}$ and it is fairly well understood. Figure 1(a) represents an schematic view of the third $\mathrm{H}$ atom trajectory towards an $\mathrm{H}_{2}$ complex consisting of two $\mathrm{H}$ atoms with fixed positions on the Y-axis with bond distance of $0.74 \AA$. The third hydrogen atom is displaced along the vector $\mathbf{r}$ with origin in the middle of the $\mathrm{H}_{2}$ bond; the trajectory is thus defined in terms of an small angle $\alpha=1^{\circ}$ (see Fig. 1(a)) between the vector $\mathbf{r}$ and the $\mathrm{X}$-axis. Notice that this system presents two symmetrical conical intersections located on the $\mathrm{X}$-axis at a distance of $\sim 0.64 \AA$.

Figure 1(b) shows the PES for the ground $\left(E_{0}\right)$ and first excited state $\left(E_{1}\right)$ along the trajectory defined in Figure 1(a). In these calculations $E_{1}$ is obtained from constrained DFT calculations in which one electron is promoted from the HOMO to the LUMO KS states. As expected, Figure 1(b) presents a conical intersection region for $r \sim 0.64 \AA$. Figure 1(c) shows the X-component of the NACV $\mathbf{d}_{i j}^{\alpha}$ between the HOMO and LUMO KS states for this trajectory (calculated using the Slater transition-state density, see below). As the two PES $E_{0}$ and $E_{1}$ approach each other close to the conical intersection region, the NACV between the HOMO and LUMO KS states presents a sharp increase. The shape of this peak compares very well with the results from accurate calculations for similar trajectories (see, e.g., Refs. 9, 46, and 47). The peak width agrees with both the reference $a b$ initio results of Varandas et $a l .{ }^{47}$ and the LR-TDDFT results;, 96 regarding the peak height, our result is in agreement with the ab initio result, ${ }^{47}$ while it is slightly smaller in the LR-TDDFT results. ${ }^{9,46}$ The orientation of the non-adiabatic coupling vector $\mathbf{d}_{i j}^{\alpha}$ changes sign when crossing the conical intersection region; also the magnitude of $\mathbf{d}_{i j}^{\alpha}$ is quite symmetric along the trajectory when approaching the conical intersection region from the left or from the right side. 

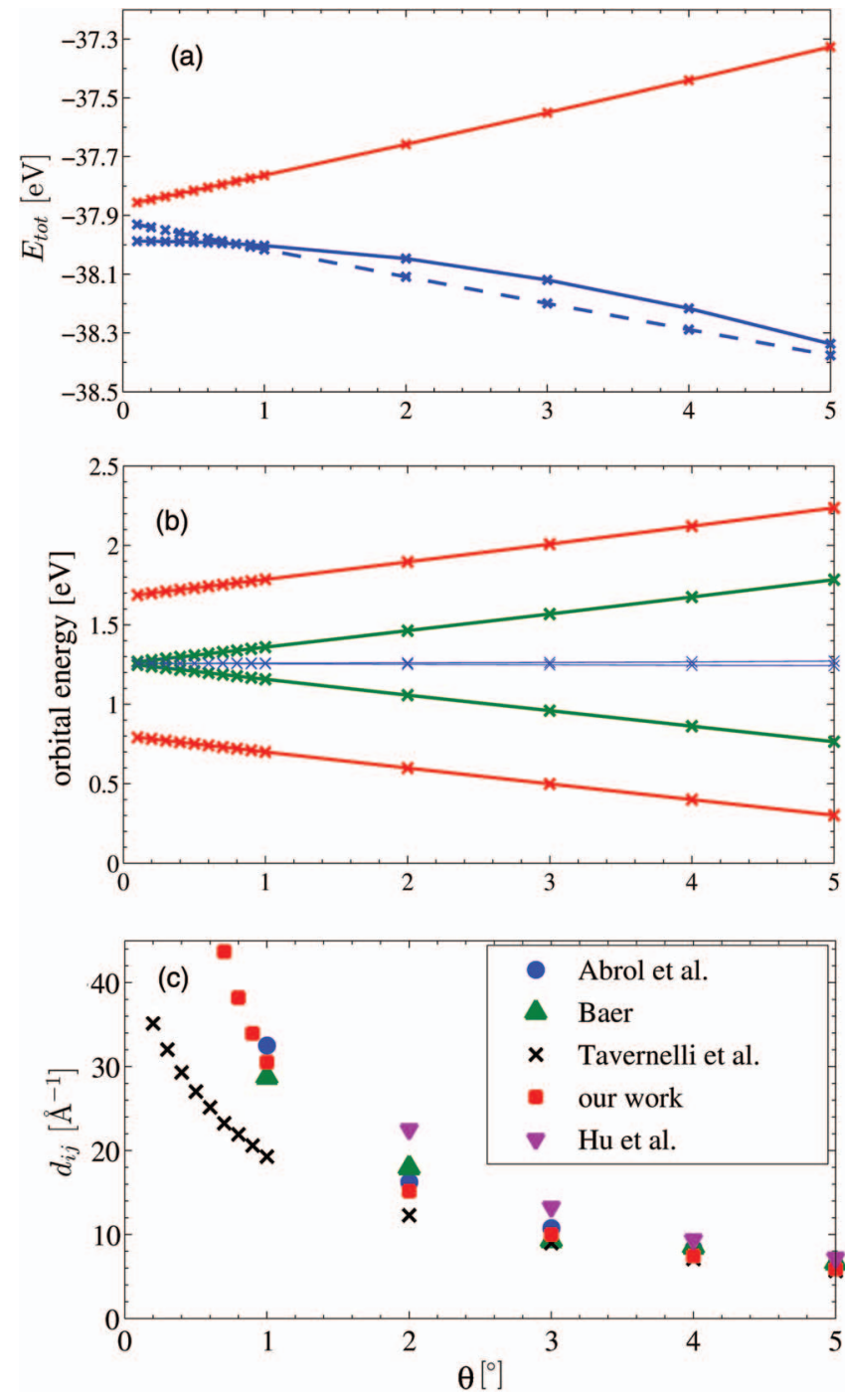

FIG. 2. (a) PES for the $\mathrm{H}_{3}$ system along the path defined by the hyperspherical coordinates $\rho=2.5 a_{0}$ and $\phi=120^{\circ}$ as a function of the angle $\theta$, see text. The ground (blue) and first excited (red) PES are shown. (b) HOMO and LUMO KS levels as a function of the angle $\theta$ for the ground state (blue), excited state (red), and Slater transition-state (green) densities. (c) NACVs ( $x$-component on the second $\mathrm{H}$ atom) as a function of $\theta$ : (circles/blue) reference ab initio results by Abrol et al. ${ }^{48}$ (taken from Ref. 46); (triangles/green) real-time TDDFT results by Baer ${ }^{46}$ (crosses/black) linear-response TDDFT results by Tavernelli et al. $9^{9}$ (triangles/violet) linear-response TDDFT results by Hu et al. ${ }^{49}$ (squares/red) our results, using a LDA Slater transition-state calculation and Eq. (18).

In order to present a detailed comparison with accurate results previously published, ${ }^{9,12,46,48}$ we have analyzed the NACVs along the path specified by the hyperspherical coordinates $^{48} \rho=2.5 a_{0}$ and $\phi=120^{\circ}$ as a function of the angle $\theta$; the Cartesian coordinates for the $3 \mathrm{H}$ atoms along this path can be found in Ref. 9. A conical intersection between the ground and first excited states is found in this path for $\theta$ $=0^{\circ}$ (i.e., the equilateral triangular geometry).

Figure 2(a) shows the PES corresponding to the LDA ground state and first excited state as a function of the angle $\theta$. In similarity to previous results using LR-TDDFT, ${ }^{9,46}$ the two PES approach each other as $\theta \rightarrow 0^{\circ}$ but are not completely degenerate at the conical intersection, $\theta=0^{\circ}$. We also find that the ground state PES presents a quadratic shape as a function of $\theta$, instead of the linear one found in the reference $a b$ initio results of Abrol et al. ${ }^{48}$ (obtained from multiconfiguration self-consistent-field configuration interaction (MC$\mathrm{SCF} / \mathrm{CI}$ ) calculations); this is due to the almost degeneracy of the HOMO and LUMO KS levels in our calculations (see Figure 2(b)) and the electronic temperature $\left(T_{e}=100 \mathrm{~K}\right)$ we have used to facilitate self-consistency. In other words, the departure from linear behavior is due to the partial occupation of the HOMO and LUMO levels in the calculation. Also shown in this figure is the PES corresponding to a constrained calculation in which the electron is forced to stay in the HOMO, recovering the expected linearity.

Since we are interested in a computationally efficient way to calculate NACVs for large systems, we analyze here the calculation of the NACVs combining Eq. (18) and the Slater transition-state method; ${ }^{7,10}$ in this approach, the manybody NACV between the ground and first excited states $\mathbf{D}_{i j}^{\alpha}$, is approximated by the single-particle NACV $\mathbf{d}_{i j}^{\alpha}$ between the HOMO and LUMO KS states calculated at the Slater transition-state density. This density is obtained in a selfconsistent KS calculation in which $1 / 2$ electron is promoted from the HOMO to the LUMO KS levels. Figure 2(b) shows the HOMO and LUMO KS levels as a function of the angle $\theta$ for the ground state, first excited state, and Slater transition-state densities. While in the ground state calculation the HOMO and LUMO levels are almost degenerate all along the path, in the excited state calculation there is an important gap between the LUMO and HOMO levels, which is as big as $\sim 0.9 \mathrm{eV}$ for the conical intersection geometry, $\theta=0^{\circ}$. In contrast, in the Slater transition-state calculation, the LUMO and HOMO levels present a linear behavior crossing at the conical intersection, in similarity to the PES obtained in accurate MCSCF/CI calculations ${ }^{48}$ (e.g., see Refs. 9 and 46). Notice that the NACV $\mathbf{d}_{i j}^{\alpha}$ in Eq. (18) depends critically on the difference between single-particle levels, $\varepsilon_{i}-\varepsilon_{j}$.

In Figure 2(c), we compare our result for the NACVs ( $x$ component on the second $\mathrm{H}$ atom ${ }^{9,46}$ ) as a function of $\theta$, using a Slater transition-state LDA-calculation and Eq. (18) for the HOMO and LUMO KS levels, with the accurate $\mathrm{MCSCF} / \mathrm{CI},{ }^{48}$ real-time TDDFT, ${ }^{46}$ and $\mathrm{LR}^{\mathrm{T}} \mathrm{TDDFT}{ }^{9,12}$ results. The agreement of our simple calculation with these accurate calculations, in particular with the reference ab initio results, ${ }^{48}$ is excellent.

The results presented for the $\mathrm{H}_{3}$ model system (Figures 1 and 2) show that using our Eq. (18) together with the Slater transition-state approximation is a very promising approach for the practical calculation of NACVs in large systems.

\section{B. Formaldimine}

We now present some calculations of the non-adiabatic coupling vectors between HOMO and LUMO KS states for the formaldimine molecule, $\mathrm{CH}_{3} \mathrm{~N}$ (see Fig. 3). This molecule has two possible symmetric conformations with the hydrogen atom bonded to the nitrogen atom placed on the left or the right with respect to the $\mathrm{C}-\mathrm{N}$ molecule axis, see Figs. 3(a) 
(a)
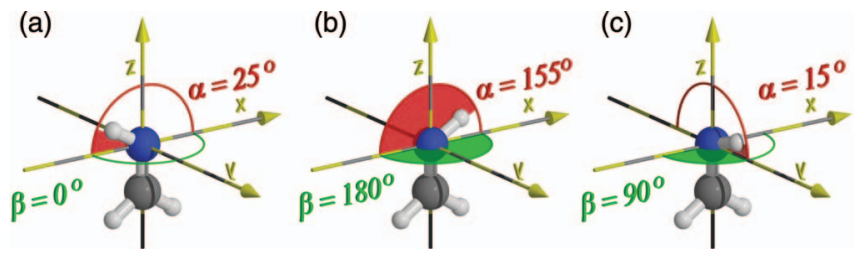

FIG. 3. The atomic structure of formaldimine corresponding to the two symmetric ground state configurations $(\mathrm{a}, \mathrm{b})$ and transition configuration (c), where $\beta=90^{\circ}$ and $\alpha \sim 15^{\circ} . \alpha$ is the angle between the XY-plane and the vector that goes from the $\mathrm{N}$ atom to the $\mathrm{H}$ atom; $\beta$ is the angle between the projection of that vector in the XY-plane and the negative $\mathrm{X}$-axis.

and 3(b). The molecule can switch between these two conformations by means of a photo-isomerisation process ${ }^{33}$ that incorporates a non-adiabatic transition between HOMO and LUMO states. In this process, the initial step is the photo-excitation of a single electron from the HOMO to LUMO orbital. After that the system evolves along the $E_{1}$ PES up to the transition region, which corresponds to the molecular structure shown in Fig. 3(c). In this region the de-excitation process might occur and the molecule geometry converges to one of the two possible ground states, Figs. 3(a) and 3(b).

In order to analyze how the non-adiabatic coupling vector $\mathbf{d}_{i j}^{\alpha}$ between HOMO and LUMO KS states evolves during this switching mechanism, we carried out a series of calculations mapping $\mathbf{d}_{i j}^{\alpha}$ as a function of the hydrogen atom position. The hydrogen atom position was systematically shifted on the hemisphere centered on the nitrogen atom with a radius of $1.15 \AA$. Thus, the hydrogen atom position is strictly given by the angles $\alpha$ and $\beta$, see Fig. 3. The angle sampling rate was $6^{\circ}$. Figure 4 shows the PES for the ground state $\left(E_{0}\right)$ and first excited state $\left(E_{1}\right)$ for the formaldimine molecule as a function of the angles $\alpha$ and $\beta$. For simplicity, in these calculations $E_{1}$ was calculated using a constrained DFT procedure maintaining the LUMO orbital occupied by a single electron and leaving a hole in the HOMO orbital.

Figure 5(a) maps the magnitude of the NACV $\left|\mathbf{d}_{i j}^{\alpha}\right|$ between the LUMO and HOMO KS states corresponding to the ground state $E_{0}$ calculation. $\left|\mathbf{d}_{i j}^{\alpha}\right|$ presents a maximum for $\alpha=12^{\circ}$ and $\beta=90^{\circ}$. The position of this maximum corresponds to the region of space where the energy differ-

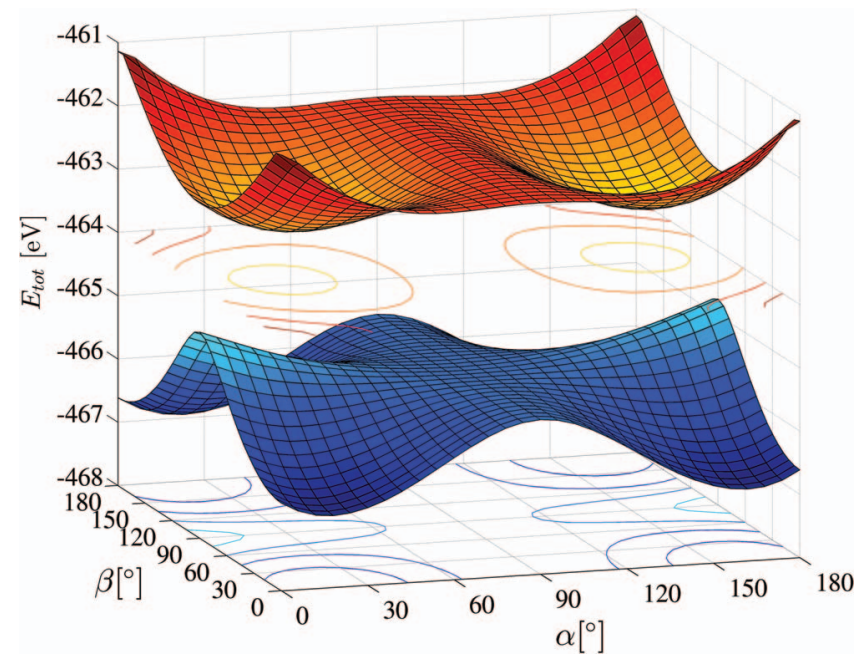

FIG. 4. Potential energy surfaces (PES) for the ground state $\left(E_{0}\right)$ and first excited state $\left(E_{1}\right)$ for the formaldimine molecule as a function of the angles $\alpha$ and $\beta$, see Fig. 3 .

ence between the PES $E_{0}$ and $E_{1}$ is minimum, $\alpha \sim 0^{\circ}-30^{\circ}$; $\beta \sim 90^{\circ}$, see Figure 4. On the other hand, the minimum of $\left|\mathbf{d}_{i j}^{\alpha}\right|$ appears for $\alpha=90^{\circ}$. This geometry corresponds to a conformation in which the $\mathrm{H}$ atom is in line with the $\mathrm{C}-\mathrm{N}$ bond, see Fig. 3.

The results shown in Figure 5(a) have been calculated for the HOMO and LUMO KS states obtained from the ground state $E_{0}$ calculation. For comparison, we have also calculated the non-adiabatic coupling vector $\mathbf{d}_{i j}^{\alpha}$ for the HOMO and LUMO KS orbitals corresponding to $E_{1}$, see Figure 5(b). The dependence of $\left|\mathbf{d}_{i j}^{\alpha}\right|$ on the angles $\alpha$ and $\beta$ shows a similar shape as in the previous case, with the maximum now placed at $\alpha=18^{\circ}$ and $\beta=90^{\circ}$. Still some important differences, due to the different HOMO and LUMO states corresponding to the $E_{0}$ and $E_{1}$ calculations, can be easily identified; in particular, the maximum for $\left|\mathbf{d}_{i j}^{\alpha}\right|$ is larger by a factor of 3 in Figure 5(a) than in Figure 5(b). Finally, Figure 5(c) shows $\left|\mathbf{d}_{i j}^{\alpha}\right|$ between the HOMO and LUMO KS states corresponding to a Slater Transition-State calculation ${ }^{7,10}$ (i.e., a constrained DFT calculation with $1 / 2$ electron promoted from HOMO to LUMO); in this calculation the value of $\left|\mathbf{d}_{i j}^{\alpha}\right|$ is in between the values obtained in Figures 5(a) and 5(b), as expected. (a)

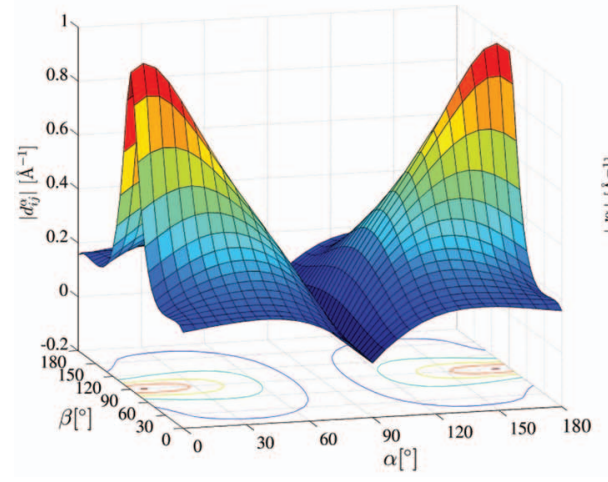

(b)

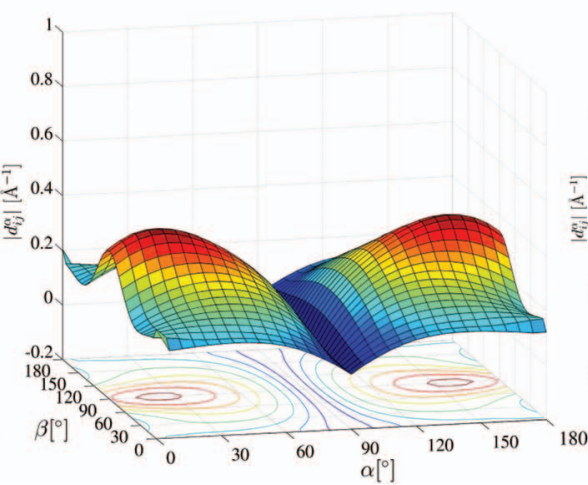

(c)

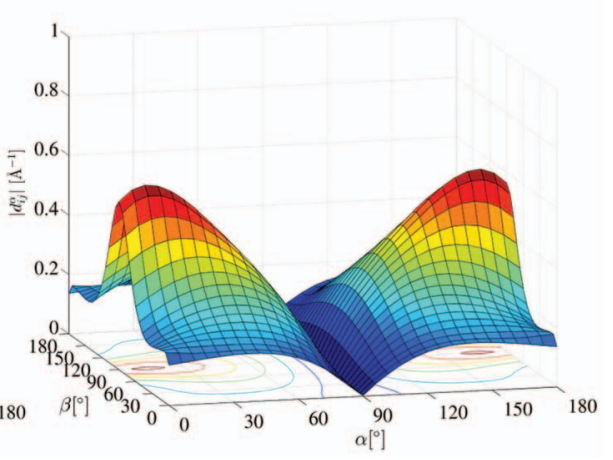

FIG. 5. $\left|\mathbf{d}_{i j}^{\alpha}\right|$ as a function of the angles $\alpha$ and $\beta$ of formaldimine molecule for the HOMO and LUMO KS states corresponding to (a) the ground state $E_{0}$ calculation; (b) the first excited state $E_{1}$ calculation; and (c) the Slater Transition State calculation. 
(a)

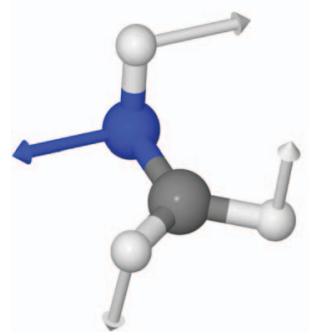

(b)

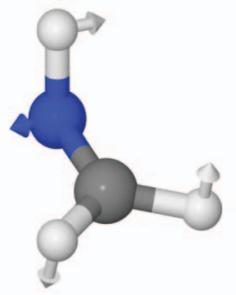

(c)

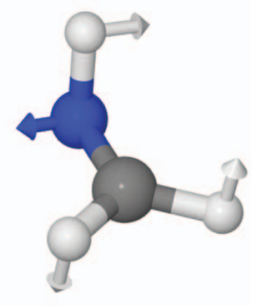

FIG. 6. NACV $\mathbf{d}_{i j}^{\alpha}$ between HOMO and LUMO KS states for the formaldimine molecule in the transition region $\left(\alpha \sim 15^{\circ}, \beta=90^{\circ}\right)$. The arrows show the direction and magnitude of the components on the different atoms $\alpha$ of the formaldimine molecule; (a) ground state, (b) excited state, and (c) Slater transition-state calculations.

Figure 6 displays a comparison for the NACVs between the HOMO and LUMO KS states corresponding to the ground state, excited state, or Slater transition-state, in the transition region (region of maximum $\left|\mathbf{d}_{i j}^{\alpha}\right|, \alpha \sim 15^{\circ}, \beta=90^{\circ}$ ). The vector orientation is very similar for all the cases with a dominant contribution on the nitrogen and the 3 hydrogen atoms. The components of the NACV on the two hydrogen atoms adjacent to the carbon atom are oriented in an anti-parallel way. The orientation of the components of $\mathbf{d}_{i j}^{\alpha}$ on the nitrogen atom and its hydrogen atom is also anti-parallel.

\section{Azobenzene}

The azobenzene molecule is composed of two phenyl rings connected by a double $\mathrm{N}=\mathrm{N}$ bond, see Fig. 7. This molecule presents two isomers, cis- and trans-azobenzene, and one of the most interesting properties of this molecule is the switching from one isomer to the other under ultraviolet

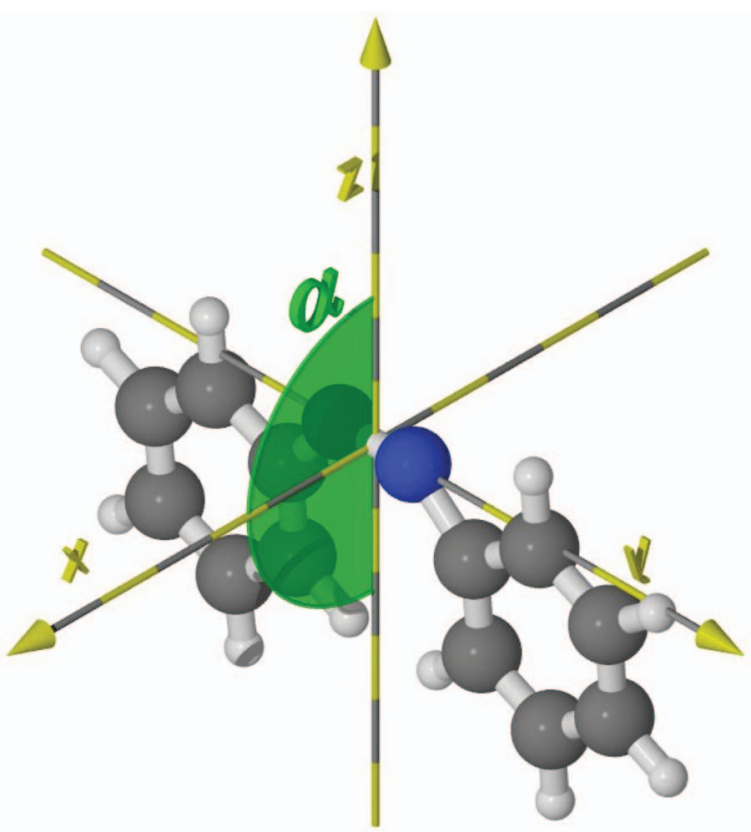

FIG. 7. The atomic structure of $c i s$-azobenzene. The angle $\alpha$ between the planes of the two phenyl rings defines the path that have been analyzed between the cis- $\left(\alpha=0^{\circ}\right.$, shown in this figure) and trans- $\left(\alpha=180^{\circ}\right)$ configurations. light (photoisomerization) or thermal excitation. These photoisomerization processes take place in the picosecond scale and the detailed mechanisms for these transitions have been under debate. ${ }^{50,51}$

In this third example, we have used Eq. (18) to calculate the NACV, $\mathbf{d}_{i j}^{\alpha}$, between the HOMO and LUMO KS states for the azobenzene molecule along a pre-selected path between the cis- and trans-conformations. In this path, we start from a geometry in which the two phenyl rings are in the same plane (see Fig. 7), which is very close to the cis-configuration; then, one of the two phenyl rings is gradually rotated along the $\mathrm{N}=\mathrm{N}$ bond so that the angle $\alpha$ between the planes of the two phenyl rings changes from $\alpha=0^{\circ}$ (cis-configuration) to $\alpha=180^{\circ}$ (trans-configuration).

Figure 8(top) shows the PES for the ground state $\left(E_{0}\right)$ and first excited state $\left(E_{1}\right)$; in similarity with the previous examples, $E_{1}$ is obtained by means of a constrained DFT calculation in which one electron from the HOMO is occupying the LUMO orbital. A conical intersection between $E_{0}$ and $E_{1}$ for $\alpha \sim 96^{\circ}$ is clearly observed. The NACV $\mathbf{d}_{i j}^{\alpha}$ between the HOMO and LUMO KS states presents a sharp increase around the conical intersection; the larger contributions to $\mathbf{d}_{i j}^{\alpha}$ are found in the $\mathrm{N}$ atoms and in the $\mathrm{C}$ atoms bonded to the $\mathrm{N}$ atoms. Figure 8(bottom) shows the modulus, $\left|\mathbf{d}_{i j}^{\alpha}\right|$, of the projection on these atoms as function of the angle $\alpha$. This
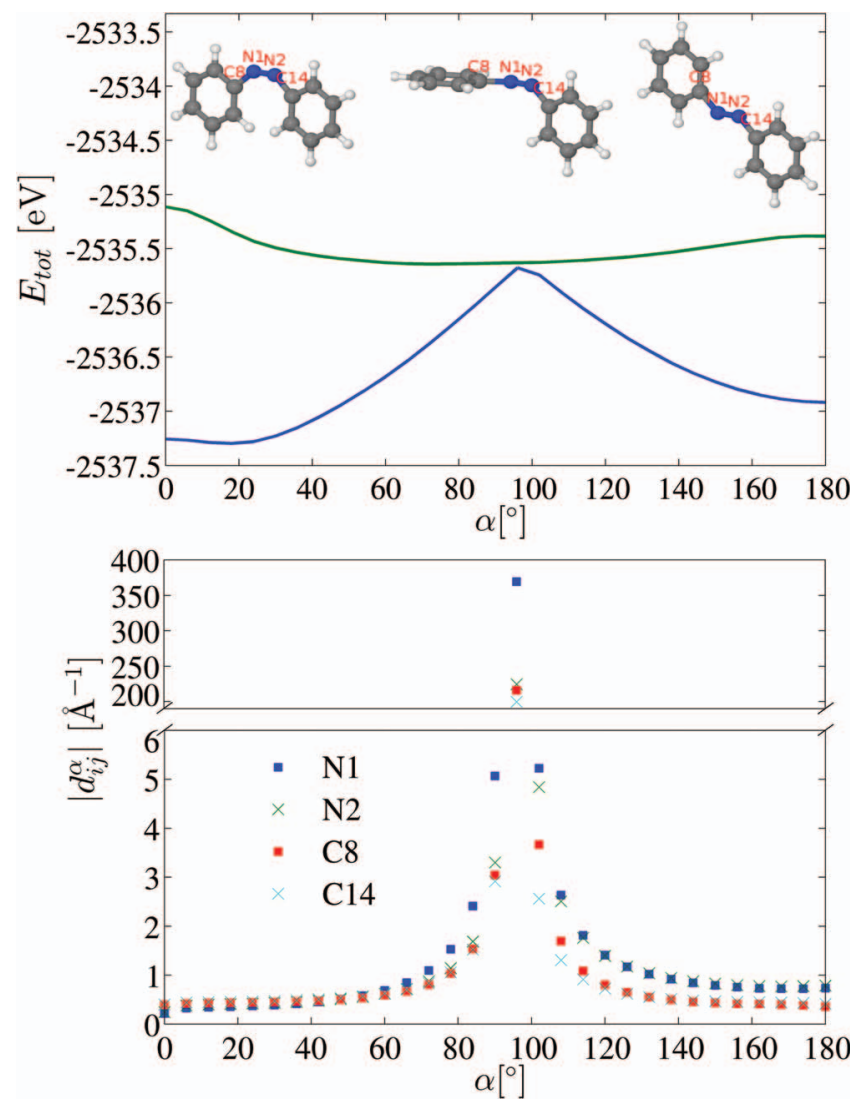

FIG. 8. (Top) Potential energy surfaces (PES) $E_{0}$ (blue) and $E_{1}$ (green) as a function of the angle $\alpha$ (see Fig. 7). (Bottom) Modulus of the projection on some atoms (N1, N2, C8, and C14, see top figure) of the non-adiabatic coupling vector between the HOMO and LUMO KS states along the isomerization pathway as a function of the angle $\alpha$. 
modulus is very similar on both $\mathrm{N}$ atoms ( $\mathrm{N} 1$ and $\mathrm{N} 2$ ), as well as in both $\mathrm{C}$ atoms (C8 and $\mathrm{C} 14)$.

\section{SUMMARY}

In summary, the development of practical methods for the calculation of the non-adiabatic coupling vectors is an important step in order to devise computationally efficient firstprinciples non-adiabatic molecular-dynamics techniques, to target practical applications for large systems. In this work we have analyzed the calculation of non-adiabatic coupling vectors in a local-orbital basis. The central result of this paper is Eq. (18) that allows the direct (on the fly) calculation of the non-adiabatic coupling vectors $\mathbf{d}_{i j}^{\alpha}$ in a practical way along the MD simulation. Equation (18) can be used within any local-orbital method; in particular, it is suitable for localorbital DFT MD techniques, such as the FIREBALL code used in the examples presented in Sec. IV. We have also derived an approximate expression, Eq. (19), especially suited for tightbinding MD and similar methods. Finally, we have presented some examples of the calculation of the non-adiabatic coupling vectors for a few simple systems: $\mathrm{H}_{3}$, formaldimine, and azobenzene. In particular, the analysis presented for the $\mathrm{H}_{3}$ system shows that using the Slater transition-state density in combination with Eq. (18) yields a very good approximation for the NACV between the ground and first excited state. This result shows that this approach is a very promising way for the practical calculation of non-adiabatic coupling vectors for large systems.

\section{ACKNOWLEDGMENTS}

This work was partially supported by Spanish Ministerio de Economía y Competitividad (Contract No. FIS2010-16046), the Comunidad de Madrid (Contract No. S2009/MAT-1467), the Office of Science, Basic Energy Sciences in the US Department of Energy (Grant No. DEFG02-10ER16164), the Czech Science Foundation (GAČR) (Project No. 204/10/0952), the Grant of the MŠMT of the Czech Republic (Grant No. ME 09048), and COST-CMTS Action CM1002 (CODECS). J.O. gratefully acknowledges support from the Spanish Ministerio de Ciencia e Innovación (PR2008-0027). E.A. gratefully acknowledges financial support by the Consejería de Educación de la Comunidad de Madrid and Fondo Social Europeo.

${ }^{1}$ P. Hohenberg and W. Kohn, Phys. Rev. 136, B864 (1964).

${ }^{2}$ W. Kohn and L. J. Sham, Phys. Rev. 140, A1133 (1965).

${ }^{3}$ N. L. Doltsinis and D. Marx, J. Theor. Comput. Chem. 1, 319 (2002).

${ }^{4}$ A. P. Horsfield, D. R. Bowler, H. Ness, C. G. Sánchez, T. N. Todorov, and A. J. Fisher, Rep. Prog. Phys. 69, 1195 (2006).

${ }^{5}$ E. J. McEniry, Y. Wang, D. Dundas, T. N. Todorov, L. Stella, R. P. Miranda, A. J. Fisher, A. P. Horsfield, C. P. Race, D. R. Mason, W. M. C. Foulkes, and A. P. Sutton, Eur. Phys. J. B 77, 305 (2010).

${ }^{6}$ C. P. Race, D. R. Mason, M. W. Finnis, W. M. C. Foulkes, A. P. Horsfield, and A. P. Sutton, Rep. Prog. Phys. 73, 116501 (2010).

${ }^{7}$ S. R. Billeter and A. Curioni, J. Chem. Phys. 122, 034105 (2005).

${ }^{8}$ D. F. Coker and L. Xiao, J. Chem. Phys. 102, 496 (1995).

${ }^{9}$ I. Tavernelli, E. Tapavicza, and U. Rothlisberger, J. Chem. Phys. 130, 124107 (2009).
${ }^{10}$ S. R. Billeter and D. Egli, J. Chem. Phys. 125, 224103 (2006).

${ }^{11}$ V. Chernyak and S. Mukamel, J. Chem. Phys. 112, 3572 (2000).

${ }^{12}$ C. Hu, O. Sugino, H. Hirai, and Y. Tateyama, Phys. Rev. A 82, 062508 (2010).

${ }^{13}$ I. Tavernelli, E. Tapavicza, and U. Rothlisberger, J. Mol. Struct.: THEOCHEM 914, 22 (2009).

${ }^{14}$ M. Tommasini, V. Chernyak, and S. Mukamel, Int. J. Quantum Chem. 85, 225 (2001).

${ }^{15}$ M. Wohlgemuth, V. Bonai-Koutecký, and R. Mitri, J. Chem. Phys. 135, 054105 (2011).

${ }^{16}$ M. Böckmann, N. L. Doltsinis, and D. Marx, J. Phys. Chem. A 114(2), 745 (2010).

${ }^{17}$ M. Böckmann, D. Marx, C. Peter, L. Delle Site, K. Kremer, and N. L. Doltsinis, Phys. Chem. Chem. Phys. 13, 7604 (2011).

${ }^{18}$ O. F. Sankey and D. J. Niklewski, Phys. Rev. B 40, 3979 (1989).

${ }^{19}$ S. Hammes-Schiffer and J. C. Tully, J. Chem. Phys. 101, 4657 (1994).

${ }^{20}$ C. Craig, W. Duncan, and O. Prezhdo, Phys. Rev. Lett. 95, 163001 (2005).

${ }^{21}$ T. W. Keal, A. Koslowski, and W. Thiel, Theor. Chem. Acc. 118, 837 (2007).

${ }^{22}$ M. J. Bearpark, M. A. Robb, and H. B. Schlegel, Chem. Phys. Lett. 223, 269 (1994).

${ }^{23}$ M. R. Manaa and D. R. Yarkony, J. Chem. Phys. 99, 5251 (1993).

${ }^{24}$ D. R. Yarkony, J. Phys. Chem. A 108, 3200 (2004).

${ }^{25}$ M. F. Herman, J. Chem. Phys. 81, 754 (1984).

${ }^{26}$ J. C. Tully, J. Chem. Phys. 93, 1061 (1990).

${ }^{27}$ O. V. Prezhdo and P. J. Rossky, J. Chem. Phys. 107, 825 (1997).

${ }^{28}$ J. P. Lewis, P. Jelínek, J. Ortega, A. A. Demkov, D. G. Trabada, B. Haycock, H. Wang, G. Adams, J. K. Tomfohr, E. Abad, H. Wang, and D. A. Drabold, Phys. Status Solidi B 248, 1989 (2011); P. Jelínek, H. Wang, J. P. Lewis, O. F. Sankey, and J. Ortega, Phys. Rev. B 71, 235101 (2005); J. P. Lewis, K. R. Glaesemann, G. A. Voth, J. Fritsch, A. A. Demkov, J. Ortega, and O. F. Sankey, ibid. 64, 195103 (2001); A. A. Demkov, J. Ortega, O. F. Sankey, and M. P. Grumbach, ibid. 52, 1618 (1995).

${ }^{29}$ Time-Dependent Density Functional Theory, Lecture Notes in Physics Vol. 706, edited by M. A. L. Marques et al. (Springer, Berlin Heidelberg, 2006).

${ }^{30}$ C. M. Isborn, X. Li, and J. C. Tully, J. Chem. Phys. 126, 134307 (2007).

${ }^{31}$ A. Castro, M. Isla, J. I. Martínez, and J. A. Alonso, Chem. Phys. 399, 130 (2012).

${ }^{32}$ S. Meng and E. Kaxiras, J. Chem. Phys. 129, 054110 (2008).

${ }^{33}$ N. Doltsinis and D. Marx, Phys. Rev. Lett. 88, 166402 (2002).

${ }^{34}$ E. Tapavicza, I. Tavernelli, and U. Rothlisberger, Phys. Rev. Lett. 98, 023001 (2007).

${ }^{35}$ J. C. Tully and R. K. Preston, J. Chem. Phys. 55, 562 (1971).

${ }^{36}$ C. E. Crespo-Hernández, B. Cohen, P. M. Hare, and B. Kohler, Chem. Rev. 104, 1977 (2004).

${ }^{37}$ E. Collini, C. Y. Wong, K. E. Wilk, P. M. G. Curmi, P. Brumer, and G. D. Scholes, Nature (London) 463, 644 (2010).

${ }^{38}$ I. Goikoetxea, J. Beltrn, J. Meyer, J. I. Juaristi, M. Alducin, and K. Reuter, New J. Phys. 14, 013050 (2012).

${ }^{39}$ P. V. Parandekar and J. C. Tully, J. Chem. Phys. 122, 094102 (2005).

${ }^{40}$ M. Elstner, D. Porezag, G. Jungnickel, J. Elsner, M. Haugk, T. Frauenheim, S. Suhai, and G. Seifert, Phys. Rev. B 58, 7260 (1998).

${ }^{41}$ M. A. Basanta, Y. J. Dappe, P. Jelínek, and J. Ortega, Comput. Mater. Sci. 39, 759 (2007).

${ }^{42}$ W. R. Duncan, C. F. Craig, and O. V. Prezhdo, J. Am. Chem. Soc. 129, 8528 (2007).

${ }^{43}$ B. F. Habenicht and O. V. Prezhdo, Phys. Rev. Lett. 100, 197402 (2008).

${ }^{44}$ S. V. Kilina, D. S. Kilin, and O. V. Prezhdo, ACS Nano 3, 93 (2009).

${ }^{45}$ S. A. Fischer, B. F. Habenicht, A. B. Madrid, W. R. Duncan, and O. V. Prezhdo, J. Chem. Phys. 134, 024102 (2011).

${ }^{46}$ R. Baer, Chem. Phys. Lett. 364, 75 (2002).

${ }^{47}$ A. J. C. Varandas, F. B. Brown, C. A. Mead, D. G. Truhlar, and N. C. Blais, J. Chem. Phys. 86, 6258 (1987).

${ }^{48}$ R. Abrol, A. Shaw, A. Kuppermann, and D. R. Yarkony, J. Chem. Phys. 115, 4640 (2001).

${ }^{49}$ C. P. Hu, H. Hirai, and O. Sugino, J. Chem. Phys. 127, 064103 (2007).

${ }^{50}$ A. Cembran, F. Bernardi, M. Garavelli, L. Gagliardi, and G. Orlandi, J. Am. Chem. Soc. 126, 3234 (2004).

${ }^{51}$ C.-W. Jiang, R.-H. Xie, F.-L. Li, and R. E. Allen, J. Phys. Chem. A 115, 244 (2011). 